MODELING, IDENTIFICATION AND CONTROL, 1994, VOL. 15, NO. 4, 253-262

doi:10.4173/mic.1994.4.4

\title{
Generalized predictive control of nonlinear systems of the Hammerstein form
}

\author{
W. WANG $\dagger$ and R. HENRIKSEN $\ddagger$
}

Keywords: Nonlinear systems, Hammerstein models, predictive control, adaptive control, stability analysis.

\begin{abstract}
A nonlinear generalized predictive control algorithm based upon a Hammerstein model is presented. Stability of the closed-loop system is analyzed with a control horizon equal to one. An adaptive nonlinear generalized predictive control algorithm with a linear estimator is then proposed. Finally, some results from simulation experiments are presented in order to show the algorithm's ability.
\end{abstract}

\section{Introduction}

Generalized predictive control (GPC) based upon linear models has enjoyed growing attention in the last few years, see, e.g., Clarke et al. (1987), Clark and Mohtadi (1989), De Keyser and Van Cauwenberghe (1985), De Keyser et al. (1988), Krämer and Unbehauen (1988), Lelic and Zarrop (1987), Wang and Henriksen (1992a, 1992b), and Ydstie (1985). Experimental studies and practical applications have demonstrated that satisfactory control performance can be obtained using GPC. However, most plants and systems to be controlled have some kind of nonlinearity, so there is definitely need to extend GPC design methods to nonlinear systems.

One extension of that kind appears in Zhu et al.(1991) where a GPC algorithm was used to control a plant described by a Hammerstein model. Due to the fact that the linear and non-linear parts of the system were considered separately in the latter, the stability of the closed-loop system was hard to analyze. Besides, a nonlinear estimation scheme had to be used in their algorithm. In this paper we will derive a nonlinear GPC algorithm based upon a Hammerstein model of the underlying system. Somewhat different from the work presented in Zhu et al. (1991) we will use a new cost function for the controller design. Stability analysis of the closed-loop system will be carried out with the control horizon equal to one. An adaptive nonlinear GPC algorithm with a linear estimation scheme will then be proposed, and some results from simulation experiments are presented at the end.

\section{Controller design}

The plant which is about to be controlled is assumed to be representable by a Hammerstein model of the form

$$
A y_{t}=B x_{t-1}+C \omega \omega_{t} / \Delta
$$

Received 6 May 1994.

†Department of Automatic Control, Northeast University of Technology, Shenyang, 110006 P.R. China.

†Department of Engineering Cybernetics, The Norwegian Institute of Technology, N-7034 Trondheim, Norway, Email: henrik @itk. unit.no.

This paper was presented at the European Control Conference, Groningen, June 28-1 July, 1993. 
where $A, B, C$ and $\Delta$ are polynomials in the backward shift operator $z^{-1}$ of the form

$$
\begin{aligned}
& A=1+a_{1} z^{-1}+\ldots+a_{n} z^{-n} \\
& B=b_{0}+b_{1} z^{-1}+\ldots+b_{m} z^{-m} \\
& C=1+c_{1} z^{-1}+\ldots+c_{l} z^{-1} \quad \Delta=1-z^{-1}
\end{aligned}
$$

whereas the static nonlinearity is given by

$$
x_{t}=r_{0}+r_{1} u_{t}+r_{2} u_{t}^{2}+\ldots+r_{p} u_{t}^{p}
$$

where $p$ is an odd number. $\left\{u_{t}\right\}$ and $\left\{y_{t}\right\}$ are the input and output processes, respectively. The model form depicted in (1) has the advantage that the controller derived in what follows will contain an integrator. $\left\{\omega_{t}\right\}$ is a stochastic process defined on a probability space $(\Omega, \mathscr{F}, \mathscr{P})$ on which we have a sequence $\left(\mathscr{F}_{t}, t \in N\right)$ of increasing $\sigma$-algebras where $\mathscr{F}_{t}$ is generated by the observations up to and including $t$. The process $\left\{\omega_{t}\right\}$ is assumed to satisfy, with probability 1 ,

$$
\begin{gathered}
E\left\{\omega_{t} \mid \mathscr{F}_{t-1}\right\}=0 \quad E\left\{\omega_{t}^{2} \mid \mathscr{F}_{t-1}\right\}=\sigma^{2} \\
\lim _{n \rightarrow \infty} \sup \left(\frac{1}{N} \sum_{t=1}^{N} \omega_{t}^{2}\right)<\infty
\end{gathered}
$$

The cost function has the following form

$$
J=E\left\{\sum_{j=1}^{N_{1}}\left[\left(y_{t+j}-y_{t+j}^{r}\right)^{2}+\lambda\left(\Delta u_{t+j-1}^{p}\right)^{2}\right] \mid \mathscr{F}_{t}\right\}
$$

where $\left\{y_{t}^{r}\right\}$ is a (known) bounded set-point sequence, $N_{1}$ is the prediction horizon whereas $\lambda$ is a weighting constant. The conditional expectation in (5) is, as indicated, taken given data up to and including time $t$. The cost on $\Delta u_{t}^{p}$ is physically meaningful because $\Delta u_{t}^{p}$ is monotically decreasing. The above therefore allows us to penalize changes in the control action.

We will, for the sake of simplicity, now assume $C=1$. It should be noted, however, that our method can readily cope with coloured noise. Now, using the following two polynomial identities

$$
1=F_{j} A \Delta+z^{-j} G_{j} \quad B F_{j}=E_{j}+z^{-j} H_{j}
$$

for $j=1,2, \ldots, N_{1}$ and where

$$
\begin{aligned}
& F_{j}=1+f_{1} z^{-1}+\ldots+f_{j-1} z^{-j+1} \\
& G_{j}=g_{0}^{j}+g_{1}^{j} z^{-1}+\ldots+g_{n}^{j} z^{-n} \\
& E_{j}=e_{0}+e_{1} z^{-1}+\ldots+e_{j-1} z^{-j+1} \\
& H_{j}=h_{0}^{j}+h_{1}^{j} z^{-1}+\ldots+h_{m-1}^{j} z^{-m+1}
\end{aligned}
$$

we can write the plant equation (1) in the form

$$
y_{t+j}=E_{j} \Delta x_{t+j-1}+G_{j} y_{t}+H_{j} \Delta x_{t-1}+F_{j} \omega_{t+j}
$$

for $j=1,2, \ldots, N_{1}$.

Using (3) we can write the $N_{1}$ equations in (8) in vector form, viz.

$$
\mathbf{y}=\mathbf{E} \sum_{i=1}^{p} r_{i} \mathbf{u}_{i}+\mathbf{G} y_{t}+\mathbf{H} \sum_{i=1}^{p} r_{i} \Delta u_{t-1}^{i}+\mathbf{F}
$$


where

Define

$$
\begin{aligned}
& \mathbf{y}=\left[y_{t+1} y_{t+2} \ldots y_{t+N_{1}}\right]^{\mathrm{T}} \\
& \mathbf{u}_{i}=\left[\Delta u_{t}^{i} \Delta u_{t+1}^{i} \ldots \Delta u_{t+N_{1}-1}^{i}\right]^{\mathrm{T}} \\
& \mathbf{G}=\left[G_{1} \ldots G_{N_{1}}\right]^{\mathrm{T}} \quad \mathbf{H}=\left[H_{1} \ldots H_{N_{1}}\right]^{\mathrm{T}} \\
& \mathbf{F}=\left[F_{1} \omega_{t+1} \ldots F_{N_{1}} \omega_{t+N_{1}}\right]^{\mathrm{T}} \\
& \mathbf{E}=\left[\begin{array}{rrrrr}
e_{0} & & & \\
e_{1} & e_{0} & & \\
e_{2} & e_{1} & e_{0} & & \\
\cdot & \cdot & \cdot & & \\
e_{N_{1}-1} & e_{N_{1}-2} & . & e_{0}
\end{array}\right]
\end{aligned}
$$

$$
\mathbf{y}_{r}=\left[y_{t+1}^{r} y_{t+2}^{r} \ldots y_{t+N_{1}}^{r}\right]^{\mathrm{T}}
$$

From the definitions above we can write (5) as

$$
J=E\left\{\left(\mathbf{y}-\mathbf{y}_{r}\right)^{\mathrm{T}}\left(\mathbf{y}-\mathbf{y}_{r}\right)+\lambda \mathbf{u}_{p}^{\mathrm{T}} \mathbf{u}_{p} \mid \mathscr{F}_{t}\right\}
$$

Substituting (9) into (12), differentiating $J$ wrt. $\mathbf{u}_{p}$, and putting the result equal to zero yield

$$
\left(r_{p} \mathbf{I}+\sum_{i=1}^{p-1} r_{i} \frac{\partial \mathbf{u}_{i}^{\mathrm{T}}}{\partial \mathbf{u}_{p}}\right) \mathbf{E}^{\mathrm{T}}\left(\mathbf{E} \sum_{i=1}^{p} r_{i} \mathbf{u}_{i}+\mathbf{G} y_{t}+\mathbf{H} \sum_{i=1}^{p} r_{i} \Delta u_{t-1}^{i}-\mathbf{y}_{r}\right)+\lambda \mathbf{u}_{p}=0
$$

Neglecting the dependence of $u_{i}(i=1, \ldots, p-1)$ on $\mathbf{u}_{p}$ we obtain

$$
r_{p} \mathbf{E}^{\mathrm{T}}\left(\mathbf{E} \sum_{i=1}^{p} r_{i} \mathbf{u}_{i}+\mathbf{G} y_{t}+\mathbf{H} \sum_{i=1}^{p} r_{i} \Delta \mathbf{u}_{t-1}^{i}-\mathbf{y}_{r}\right)+\lambda \mathbf{u}_{p}=0
$$

which can be written as

$$
r_{p} \mathbf{E}^{\mathrm{T}} \mathbf{E} \sum_{i=1}^{p-1} r_{i} \mathbf{u}_{i}+\left(r_{p}^{2} \mathbf{E}^{\mathrm{T}} \mathbf{E}+\lambda \mathbf{I}\right) \mathbf{u}_{p}=r_{p} \mathbf{E}^{\mathrm{T}}\left(\mathbf{y}_{r}-\mathbf{G} y_{t}-\mathbf{H} \sum_{i=1}^{p} r_{i} \Delta u_{t-1}^{i}\right)
$$

(15) constitutes a total of $N_{1}$ equations with $N_{1}$ unknowns. It is not, however, easy to find a solution for $u_{t}$. In the above, note that the control horizon and the output prediction horizon have been selected to to be the same, i.e., $N_{1}$. This is not a necessary requirement. A control horizon $N_{u}<N_{1}$ can be used, see Clarke et al. (1987). Furthermore, it is possible to obtain satisfactory control of most plants by putting the control horizon $N_{u}=1$, see again Clarke et al. (1987). We therefore adopt $N_{u}=1$ in our design of the controller, i.e., $\Delta u_{t+j}^{i}=0$ for $j=1, \ldots, N_{1}-1$. In this case $\mathbf{u}_{i}$ and $\mathbf{E}$ as defined by (10) will take the forms, respectively,

$$
\mathbf{u}_{i}=\Delta u_{t}^{i} \quad \mathbf{E}=\left[e_{0} e_{1} \ldots e_{N_{1}-1}\right]^{\mathrm{T}}
$$

From (16) and the fact that $z^{-1} u_{t}^{i}=u_{t-1}^{i}$ for $i=1, \ldots$, we can rewrite (15) as

$$
k e \sum_{i=1}^{p-1} r_{i} u_{t}^{i}+u_{t}^{p}=P y_{t+N_{1}}^{r}-\alpha y_{t}-\beta \sum_{i=1}^{p} r_{i} \Delta u_{t-1}^{i}+k e \sum_{i=1}^{p-1} r_{i} u_{t-1}^{i}+u_{t-1}^{p}
$$

where

$$
k=\frac{r_{p}}{r_{p}^{2} e+\lambda} e=\sum_{i=0}^{N_{1}-1} e_{i}^{2}
$$


whereas $P, \alpha$, and $\beta$ are polynomials in the backward shift operator $z^{-1}$ given by $\left(G_{j}\right.$ and $H_{j}$ are polynomials, see (7))

$$
\begin{gathered}
P=k\left(e_{N_{1}-1}+e_{N_{1}-2} z^{-1}+\ldots+e_{0} z^{-N_{1}+1}\right) \\
\alpha=k \sum_{j=1}^{N_{1}} e_{j-1} G_{j} \quad \beta=k \sum_{j=1}^{N_{1}} e_{j-1} H_{j}
\end{gathered}
$$

Equation (17) is a p'th order Hammerstein polynomial in $u_{t}$ which is fairly easy to solve numerically in order to find $u_{t}$. For example, the improved root solving method given in Zhu et al. (1991) can be used. Note that a real root of minimum magnitude can always be found because $p$ is odd.

\section{Stability analysis}

Let us rewrite (17) in the form

$$
\left(k e+z^{-1} \beta\right) \sum_{i=1}^{p-1} r_{i} \Delta u_{t}^{i}+\left(1+z^{-1} r_{p} \beta\right) \Delta u_{t}^{p}=P y_{t+N_{1}}^{r}-\alpha y_{t}
$$

From (1) and (3) we have

$$
A \Delta y_{t}=z^{-1} B \sum_{i=1}^{p-1} r_{i} \Delta u_{t}^{i}+z^{-1} r_{p} B \Delta u_{t}^{p}+\omega_{t}
$$

Lemma

Let a system be described by ( $T$ is a polynomial in $z^{-1}$ )

$$
T \Delta u_{t}^{p}=A y_{t+d}+B \sum_{j=1}^{p-1} r_{j} \Delta u_{t}^{j}+C \omega_{t}
$$

and let $\omega_{t}$ satisfy (4). If $T$ is stable, then for some positive integer $d$

$$
\frac{1}{N} \sum_{t=1}^{N}\left(\Delta u_{t}^{p}\right)^{2} \leqslant \frac{K_{1}}{N} \sum_{t=1}^{N} y_{t+d}^{2}+K_{2}
$$

where $0<K_{1}<\infty$ and $0<K_{2}<\infty$.

Proof. See the Appendix.

\section{Theorem}

If the control law given by (17) is used with $N_{1}$ and $\lambda$ chosen such that the polynomial

$$
T=A \Delta\left(1+z^{-1} r_{p} \beta\right)+z^{-1} r_{p} \alpha B
$$

is stable, then with probability 1

(1) The resulting closed-loop system will be stable in the sense that $\left\{\Delta u_{t}^{i}\right\}$, $i=1, \ldots, p$, and $\left\{y_{t}\right\}$ are sample mean square bounded.

(2) The control law (17) minimizes the cost function

$$
J^{\prime}=E\left\{\left[P\left(y_{t+N_{1}}-y_{t+N_{1}}^{r}\right)+\lambda^{\prime} \Delta u_{t}^{p}\right]^{2} \mid \mathscr{F}_{t}\right\}
$$

where $\lambda^{\prime}=\lambda k / r_{p}$. Moreover, the minimum possible value of the quadratic cost function (25) is

$$
\gamma^{2}=k^{2} \sigma^{2} \sum_{j=1}^{N_{1}}\left(\sum_{i=0}^{N_{1}-j} f_{i} e_{i+j-1}\right)^{2}
$$


(3) For constant reference $y_{t}^{r}$ and with $\omega_{t}=0$ it follows that

$$
\lim _{t \rightarrow \infty}\left(y_{t}-y_{t}^{r}\right)=0
$$

Proof. (1) Multiplying (20) by $A \Delta$ and $z^{-1} r_{p} B$ respectively and using (21) we obtain

$$
\begin{gathered}
T \Delta u_{t}^{p}=A \Delta P y_{t+N_{1}}^{r}-\left[A \Delta\left(k e+z^{-1} \beta\right)+z^{-1} \alpha B\right] \sum_{i=1}^{p-1} r_{i} \Delta u_{t}^{i}-\alpha \omega_{t} \\
T y_{t}=z^{-1} r_{p} B P y_{t+N_{1}}^{r}+z^{-1} \frac{\lambda}{r_{p}} k B \sum_{i=1}^{p-1} r_{i} \Delta u_{t}^{i}+\left(1+z^{-1} r_{p} \beta\right) \omega_{t}
\end{gathered}
$$

If $T$ is stable, then conclusion (1) of the theorem follows from (28) and (29), use of superposition, the above lemma, the last of the assumptions in (4), and boundedness of $\left\{y_{t}^{r}\right\}$.

(2) Multiplying (9) by $r_{p} \mathbf{E}^{\mathrm{T}}$, adding $\lambda \Delta u_{i}^{p}$ on both sides, and then using (16) we have

$$
r_{p} \mathbf{E}^{\mathrm{T}} \mathbf{y}+\lambda \Delta u_{t}^{p}=r_{p}^{2} e \Delta u_{t}^{p}+\lambda \Delta u_{t}^{p}+r_{p} e \sum_{i=1}^{p-1} r_{i} \Delta u_{t}^{i}+r_{p} \mathbf{E}^{\mathrm{T}}\left(\mathbf{G} y_{t}+\mathbf{H} \sum_{i=1}^{p} r_{i} \Delta u_{t-1}^{i}+\mathbf{F}\right)
$$

which results in

$$
\Delta u_{t}^{p}=P y_{t+N_{1}}+\lambda^{\prime} \Delta u_{i}^{p}-v_{t+N_{1}}-\alpha y_{t}-\beta \sum_{i=1}^{p} r_{i} \Delta u_{t-1}^{i}-k e \sum_{i=1}^{p-1} r_{i} \Delta u_{t}^{i}
$$

where

$$
v_{t+N_{1}}=k \sum_{j=1}^{N_{1}} e_{j=1} F_{j} \omega_{r+j}
$$

Defining

$$
\phi_{t+N_{1}}=P y_{t+N_{1}}+\lambda^{\prime} \Delta u_{t}^{p}
$$

we can write (31) as

$$
\phi_{t+N_{1}}-v_{t+N_{1}}=\alpha y_{t}+\beta \sum_{i=1}^{p} r_{i} \Delta u_{t-1}^{i}+\Delta u_{t}^{p}+k e \sum_{i=1}^{p-1} r_{i} \Delta u_{t}^{i}
$$

Here we note that $\phi_{t+N_{1}}-v_{t+N 1}$ is $\mathscr{F}_{t}$-measurable. It is obvious that $\phi_{t+N_{1}}-v_{t+N_{1}}$ is the optimal linear prediction of $\phi_{t+N_{1}}$ given $\mathscr{F}_{t}$, i.e.,

$$
\phi_{t+N_{1}}^{0}=\phi_{t+N_{1}}-v_{t+N_{1}}=\alpha y_{t}+\beta \sum_{i=1}^{p} r_{i} \Delta u_{t-1}^{i}+\Delta u_{t}^{p}+k e \sum_{i=1}^{p-1} r_{i} \Delta u_{t}^{i}
$$

Now, making use of the fact that $\phi_{t+N_{1}}=\phi_{t+N_{1}}^{0}+v_{t+N_{1}}$ and then substituting this equation and (33) into (25), we obtain after some manipulations

$$
J^{\prime}=E\left\{\left(\phi_{t+N_{1}}^{0}-P y_{t+N_{1}}^{r}\right)^{2} \mid \mathscr{F}_{t}\right\}+E\left\{v_{t+N_{1}}^{2} \mid \mathscr{F}_{t}\right\} \geqslant E\left\{v_{t+N_{1}}^{2} \mid \mathscr{F}_{t}\right\}
$$

The first term in the middle part of (36) is greater than or equal to zero. It becomes equal to zero by putting

$$
\phi_{t+N_{1}}^{0}=P y_{t+N_{1}}^{r}
$$

Substituting (35) onto (37) yields the control law defined by (17). Finally, from (32) we obtain

$$
E\left\{v_{t+N_{1}}^{2} \mid \mathscr{F}_{t}\right\}=E\left\{\left(k \sum_{j=1}^{N_{1}} e_{j-1} \sum_{i=0}^{j-1} f_{i} \omega_{t+j-i}\right)^{2} \mid \mathscr{F}_{t}\right\}=k^{2} \sigma^{2} \sum_{j=1}^{N_{1}}\left(\sum_{i=0}^{N_{1}-j} f_{i} e_{i+j-1}\right)^{2}=\gamma^{2}
$$


(3) Using (6) and the definitions of the polynomials $\alpha$ and $\beta$, we can write the polynomial $T$ as

$$
T=A \Delta\left(1-z^{-1} r_{p} k \sum_{i=1}^{N_{1}} z^{i} e_{i-1} E_{i}\right)+z^{-1} r_{p} k B \sum_{i=1}^{N_{1}} z^{i} e_{i-1}
$$

From (39) we find

$$
T(1)=r_{p} k B(1) \sum_{i=1}^{N_{1}} e_{i-1}=r_{p} B(1) P(1)
$$

Conclusion (3) now follows immediately from (29).

\section{The ANGPC algorithm}

We assumed in the previous section that the plant parameters were all known. When the plant parameters are unknown we will have to use a parameter estimator. In this section an adaptive nonlinear generalized predictive control algorithm (ANGPC) is defined by combining the controller derived in Section 2 with a general parameter estimation scheme, Our ANGPC algorithm is based upon the following assumptions:

A1. The polynomial degrees $n$ and $m$ in (1) are known.

A2. $p$ is a known odd positive integer.

We now rewrite the plant equation (1) in the form

$$
A^{\prime} y_{t}=\sum_{i=1}^{p} B_{i} \Delta u_{t-1}^{i}+\omega_{t}
$$

where

$$
\begin{aligned}
& A^{\prime}=A \Delta=1+a_{1}^{\prime} z^{-1}+\ldots+a_{n+1}^{\prime} z^{-n-1} \\
& B_{i}=r_{i} B=b_{0}^{i}+b_{1}^{i}+\ldots+b_{m}^{i} z^{-m}, i=1, \ldots, p
\end{aligned}
$$

Computation of the polynomial $G_{j}$ and two other polynomials $E_{i j}$ and $H_{i j}$ are done from the two following equations

$$
\begin{gathered}
1=F_{j} A^{\prime}+z^{-j} G_{j} \\
B_{i} F_{j}=E_{i j}+z^{-j} H_{i j}
\end{gathered}
$$

where

$$
\begin{aligned}
& E_{i j}=e_{0}^{i}+e_{1}^{i} z^{-1}+\ldots+e_{j-1}^{i} z^{-j+1} \\
& H_{i j}=h_{0}^{i j}+h_{1}^{i j} z^{-1}+\ldots+h_{m-1}^{i j} z^{-m+1}
\end{aligned}
$$

for $i=1, \ldots, p$ and $j=1, \ldots, N_{1}$.

Define

$$
\begin{aligned}
\mathbf{E}_{i} & =\left[e_{0}^{i} e_{1}^{i} \ldots e_{N_{1}-1}^{i}\right]^{\mathrm{T}} \\
\mathbf{H}_{i} & =\left[H_{i 1} H_{i 2} \ldots H_{i N_{1}}\right]^{\mathrm{T}}
\end{aligned}
$$

This allows us to write the control law (17) in the form

$$
\mathbf{E}_{p}^{\mathrm{T}} \sum_{i=1}^{p-1} \mathbf{E}_{i} \mathbf{u}_{i}+\left(\mathbf{E}_{p}^{\mathrm{T}} \mathbf{E}_{p}+\lambda\right) \mathbf{u}_{p}=\mathbf{E}_{p}^{\mathrm{T}}\left(\mathbf{y}_{r}-\mathbf{G} y_{t}-\sum_{i=1}^{p} \mathbf{H}_{i} \Delta u_{t-1}^{i}\right)
$$


The ANGPC algorithm now follows below.

(1) The parameters of system (41) are updated from the following estimation scheme (Goodwin et al. 1980)

$$
\begin{gathered}
\theta_{t}=\theta_{t-1}+\frac{\rho}{\Lambda_{t-1}} \mathbf{X}_{t-1}\left[y_{t}-\mathbf{X}_{t-1}^{\mathrm{T}} \theta_{t-1}\right], \rho>0 \\
\Lambda_{t}=\Lambda_{t-1}+\mathbf{X}_{t}^{\mathrm{T}} \mathbf{X}_{t}, \Lambda_{0}=1
\end{gathered}
$$

where

$$
\begin{gathered}
\mathbf{X}_{t}^{\mathrm{T}}=\left[y_{t} \ldots y_{t-n} \Delta u_{t} \ldots \Delta u_{t-m} \ldots \Delta u_{t}^{p} \ldots \Delta u_{t-m}^{p}\right] \\
\theta^{\mathrm{T}}=\left[-a_{1}^{\prime} \ldots-a_{n+1}^{\prime} b_{0}^{1} \ldots b_{m}^{1} \ldots b_{p}^{0} \ldots b_{m}^{p}\right]
\end{gathered}
$$

(2) $G_{j}, E_{i j}$, and $H_{i j}$ are calculated from (43) and (44), respectively.

(3) The control action $u_{t}$ is determined from equation (47).

Note that the above parameter estimation scheme is linear, whereas a nonlinear scheme has to be used in the algorithm introduced by Zhu et al. (1991) in order to update the parameters of both the linear part and of the nonlinear part, viz. $a_{i}, b_{i}$, and $r_{i}$, respectively.

\section{Simulation experiments}

In order to investigate the performance of the above ANGPC algorithm, we will in this section present some results obtained from simulation experiments. For the purpose of being able to compare results, we will use the same two plants ( $\mathrm{L} 1$ and $\mathrm{L} 2)$ and the same two nonlinearities (NL1 and NL2) as were used by Zhu et al. (1991). Referring to (1) and (2), the following values are used:

$$
\begin{aligned}
& \text { L1: } a_{1}=-0.9 \quad b_{0}=1 \quad b_{1}=2 \\
& \text { L2: } a_{1}=-2.87 \quad a_{2}=2.74 \quad a_{3}=-0.87 \\
& b_{0}=0.04 \quad b_{1}=0.002 \quad b_{2}=-0.037 \\
& \text { NLl: } r_{0}=1 \quad r_{1}=1 \quad r_{2}=1 \\
& r_{3}=0.2 \\
& \text { NL2: } r_{0}=0 \quad r_{1}=1 \quad r_{2}=0 \\
& r_{3}=-1
\end{aligned}
$$

Here we note that L1 is open-loop stable and nonminimum phase, whereas L2 is openloop unstable and minimum phase. In addition, $\omega_{t}$ is here a zero-mean random disturbance with covariance $\sigma^{2}=0 \cdot 1$.

In order to consider transient behaviour, we assign a set-point sequence as follows

L1: L2:

Samples: Set-point value: Samples: Set-point value:

$\begin{array}{cccc}01-20 & 1 & 01-40 & 1 \\ 21-40 & 2 & 41-80 & 2 \\ 41-60 & 1 & 081-120 & 1 \\ 61-80 & 0 & 121-160 & 0\end{array}$

The cycle from respectively 1 to 80 or from 1 to 160 is repeated periodically in each experiment. In the plots shown in Fig. 1-4 the output $y_{t}$ and the control input $u_{t}$ are shown as unbroken lines, whereas the set-point sequence $y_{t}^{r}$ and the intermediate variable $x_{r}$ are shown as broken lines. 
The parameters of the ANGPC algorithm are chosen as $N_{1}=3$ whereas $\lambda=0.01$ for $\mathbf{L} 1$ and $\lambda=0$ for $\mathbf{L} 2$. From the plots in the figures it is seen that the output tracks the setpoint sequence quite well even though there is a random disturbance. The large input and output deviations at the outset are more or less what should be expected in a commissioning period when the parameter estimates have not yet converged. The predictive nature of the controller can clearly be seen in the plots, where prior knowledge of a change in the set-point value has caused the output $y_{t}$ to start moving before the actual change in the set-point has occurred. If we compare with the simulation experiments in Zhu et al. (1991), a somewhat better performance of our algorithm is seen. Whereas rapid changes in the control signal $u_{t}$ occur in the simulation experiments of L2+NL1 and L2 + NL2 in the above reference, the control signal in our simulation experiments of the same systems (Figs. 3(b) and 4(b) appears to be quite smooth.

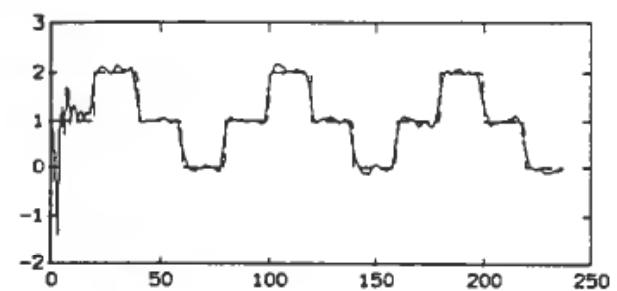

(a)

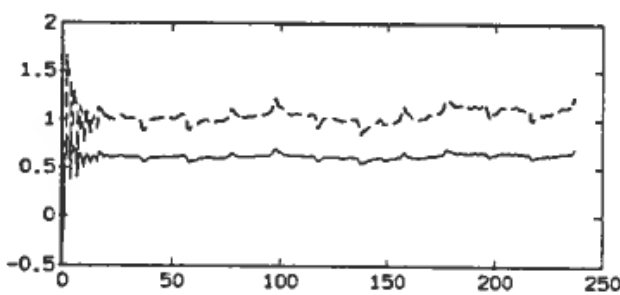

(b)

Figure 1 (a). Output and set-point of system L1 + NL1. (b) Control and intermediate variable of L1 + NL1.

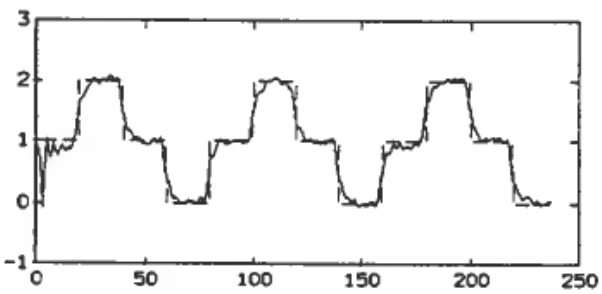

(a)

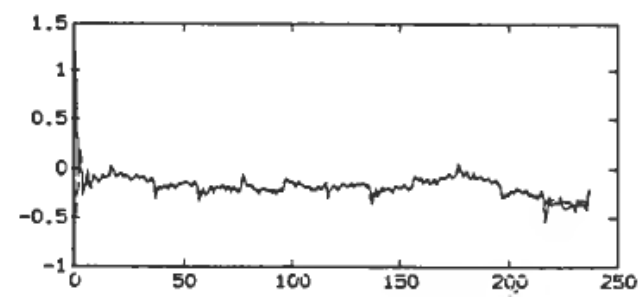

(b)

Figure 2(a). Output and set-point for system L1 + NL2. (b) Control and intermediate variable of $\mathrm{L} 1+\mathrm{NL} 2$.

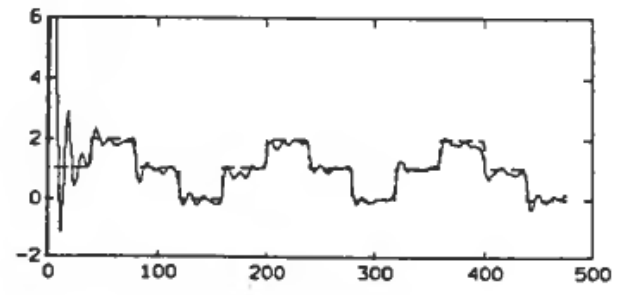

(a)

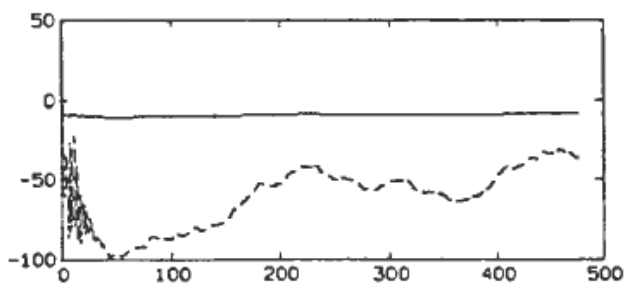

(b)

Figure 3(a). Output and set-point for system L2+NL1. (b) Control and intermediate variable of $\mathrm{L} 2+\mathrm{NL} 1$. 


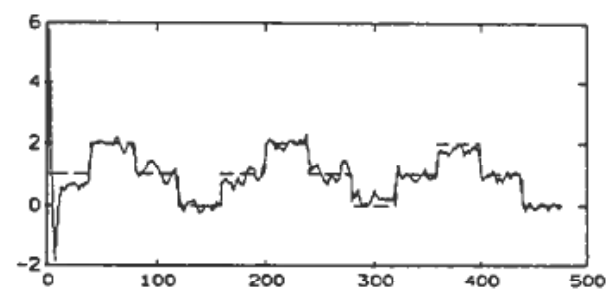

(a)

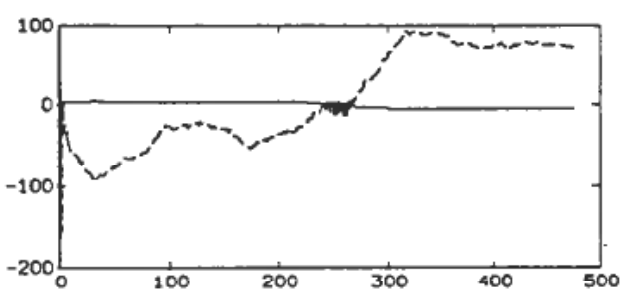

(b)

Figure 4(a). Output and set-point of system L2 + NL2.(b) Control and intermediate variable of $\mathrm{L} 2+\mathrm{NL} 2$.

\section{Conclusion}

In this paper we have derived a nonlinear generalized predictive control algorithm for systems which can be modelled by a Hammerstein model. Stability of the algorithm has, subject to fairly to weak assumptions, been shown with the control horizon $N_{u}=1$. We have also suggested an adaptive nonlinear generalized predictive control algorithm which turned out to perform quite well in simulation experiments. Stability and convergence of the latter algorithm have, however, not been shown. As point of fact, we have so far not even tried to do that because analysis of stability and convergence of nonlinear generalized predictive control algorithms in adaptive or self-tuning form is indeed a very difficult task to carry out.

\section{APPENDIX}

Proof of the Lemma

Using superposition and Lemmas A.1. and A.5 in Goodwin et al. (1981), we obtain

$$
\frac{1}{N} \sum_{t=1}^{N}\left(\Delta u_{t}^{p}\right)^{2} \leqslant \frac{L_{1}}{N} \sum_{t=1}^{N} y_{t+d}^{2}+\sum_{j=1}^{p-1} \frac{L_{2 j}}{N} \sum_{t=1}^{N}\left(\Delta u_{t}^{j}\right)^{2}+\frac{L_{3}}{N} \text { wp. } 1
$$

Define, for $j=1,2, . ., p$,

$$
\delta_{t}^{j}=\left\{\begin{array}{l}
\Delta u_{t}^{j} \text { if }\left|\Delta u_{t}^{j}\right|>M, M>0 \\
0 \text { otherwise }
\end{array}\right.
$$

We then have, for $j=1,2, \ldots, p$,

$$
\frac{1}{N} \sum_{t=1}^{N}\left(\Delta u_{t}^{j}\right)^{2} \leqslant \frac{1}{N} \sum_{t=1}^{N}\left(\delta_{t}^{j}\right)^{2}+M^{2}
$$

Take $M$ large enough such that

$$
\frac{L_{2 j}}{N} \sum_{t=1}^{N}\left(\delta_{t}^{j}\right)^{2} \leqslant \frac{1}{2(p-1) N} \sum_{t=1}^{N}\left(\Delta u_{t}^{p}\right)^{2}
$$

It then follows that

$$
\frac{1}{N} \sum_{t=1}^{N}\left(\Delta u_{t}^{p}\right)^{2} \leqslant \frac{L_{1}}{N} \sum_{t=1}^{N} y_{t+d}^{2}+\frac{1}{2 N} \sum_{t=1}^{N}\left(\Delta u_{t}^{p}\right)^{2}+L_{4} \text { wp. } 1
$$

and hence

$$
\frac{1}{N} \sum_{t=1}^{N}\left(\Delta u_{t}^{p}\right)^{2} \leqslant \frac{K_{1}}{N} \sum_{t=1}^{N} y_{t+d}^{2}+K_{2} \text { w.p. } 1
$$




\section{REFERENCES}

Clarke, D. W. (1988). Application of generalized predictive control. Proc. IF AC Symp. on Adaptive Control of Chemical Processes, Copenhagen, Denmark.

Clarke, D. W., Mohtadi C. and TufFs, P.S. (1987). Generalized predictive control-Part I and II. Automatica, 23, 137-160.

Clarke, D. W. and Mohtadi, C. (1989). Properties of generalized predictive control. Automatica, 25, 859-875.

DeKeyser, R. M. C. and VAN CAUWENBerghe, A. R. (1985). Extended prediction self-adaptive control. Proc. 7th IF AC/IFORS Symp. on Identification and System Parameter Estimation, York, UK. 1255-1260.

De Keyser, R. M., Van de Velde, Ph. G. A. and Dumortier F. A. G. (1988). A comparative study of self-adaptive long-range predictive control methods. Automatica, 24, 149-163.

Goodwin, G. C., Sin, K. S. and Saluja, K. K. (1980). Stochastic adaptive control and prediction: the general delay-coloured noise case. IEEE Trans. Automatic Control, 25, 946-950.

Goodwin, G. C., Ramadge, P. J. and Caines, P. E. (1981). Discrete time stochastic adaptive control. SIAM J. Control and Optimization, 19, 829-853.

KräMER, K. and L'VBEHAUEN, H. (1988). Survey to adaptive long-range predictive control. Proc. 12th IMACS World Congress on Scientific Computation, Paris, France, 358-363.

LeLIC, M. A. and ZARROP, M. B. (1987). Generalized pole-placement self-tuning controller-Part 1 and 2. Int. J. Control, 46, 547-601.

WANG, W. and HENRIKSEN, R. (1992a). Direct adaptive generalized predictive control. Proc. 1992 American Control Conference, Chicago, Illinois, 2402-2406.

WANG, W. and HENRIKSEN, R. (1992b). A direct adaptive generalized predictive controller and some of its global convergence properties. Proc. 4th IF AC Int. Symp. Adaptive Systems in Control and signal Processing, Grenoble, France, 187-192.

YDSTIE, B. E. (1985). Extended horizon adaptive control. Proc. 9th IF AC World Congress, Budapest, Hungary. Vol. VII, 133-137.

ZHU, Q. M., WARWICK, K. and DUUCE, J. L. (1991). Adaptive general predictive controller for nonlinear systems. IEE Proc.-D, 138, $33-40$. 